\title{
Variables de familia, aptitudes intelectuales y logro en lectura en estudiantes mexicanos
}

\section{Aldo Bazán ${ }^{1}$, Doris Castellanos ${ }^{2}$, Violeta Fajardo ${ }^{3}$}

\author{
${ }^{1}$ Universidad Nacional Federico Villarreal, Facultad de Psicología. Perú \\ ${ }^{2}$ Universidad Autónoma del Estado de Morelos, Centro de Investigación \\ Trandisciplinar en Psicología. México \\ ${ }^{3}$ Servicios Psicológicos Integrales de México SEPIMEX. México
}

\section{Perú}

México

Correspondencia: Aldo Bazán-Ramírez. Dirección postal: Jr. Mariscal Cáceres 144 José C. Mariátegui, Villa María del Triunfo. Lima, Perú.

E-mail: abazan@unfv.edu.pe ; abazanramirez@gmail.com

(C) Universidad de Almería and Ilustre Colegio Oficial de la Psicología de Andalucía Oriental (Spain)) 


\section{Resumen}

Introducción. El rendimiento académico de los estudiantes puede verse influenciado por varios factores de contexto, entre otros, por la práctica educativa de la familia de origen y el apoyo de los padres con las tareas y el aprendizaje en casa. De igual manera, el rendimiento de los alumnos puede ser influido por las características personales de los estudiantes, por ejemplo, sus habilidades intelectuales. El objetivo de este estudio fue probar un modelo explicativo de relaciones estructurales entre variables del apoyo de los padres a sus hijos en el estudio y aprendizaje en casa (autoevaluación de los padres y percepción de los hijos), las aptitudes intelectuales de los estudiantes, el nivel educativo de los padres, y el logro académico en comprensión lectora, al final de la educación primaria.

Método. Participaron 362 estudiantes de sexto grado de escuelas rurales, indígenas y públicas de Sonora y Morelos, México. El estudio se llevó a cabo en el año escolar 2015 del sistema educativo mexicano. El apoyo de los padres al aprendizaje de sus hijos en casa fue evaluado con dos versiones del Cuestionario de Percepción de Apoyo Familiar (FSPQ), una para los padres y otra para los estudiantes. El FSPQ evaluó cuatro dimensiones de apoyo: 1. Ayuda para la tarea /Revisión, 2. Comunicación con la escuela/Participación, 3. Condiciones y recursos para estudiar en el hogar, y 4. Monitoreo/Comunicación con los maestros. Para evaluar el desempeño académico en comprensión lectora se utilizó un instrumento apegado al plan de estudios para el sexto grado de la escuela primaria, con 17 ítems, que evalúan cinco dimensiones: 1. Identificación de errores ortográficos en cinco textos cortos, 2. Inferir información implícita y entender metáforas de textos narrativos y relatos cortos, 3. Reconocimiento de figuras literarias en poesías, 4. Inferir significado de palabras en lengua indígena, y 5. Identificando secciones de un periódico. Para evaluar las aptitudes intelectuales, se utilizaron versiones reducidas de dos escalas del Screening Assessment for Gifted Elementary and Middle School Students (SAGES-2), validado en el contexto mexicano: 1. Trece ítems de la escala Lengua y Literatura/Ciencias Sociales y 17 ítems de la escala de Razonamiento General.

Resultados. Los resultados de un modelo de regresión estructural con el programa EQS 6.4, mostraron que el rendimiento en lectura fue explicado principal y significativamente por las aptitudes intelectuales de los estudiantes, y, en segundo lugar, por el apoyo de los padres (evaluado por ellos mismos). Sin embargo, el apoyo familiar percibido por los estudiantes no tuvo efectos significativos en el rendimiento en lectura. Por otro lado, el nivel académico de los padres mostró un efecto significativo en las aptitudes intelectuales de los estudiantes, y tuvo también un efecto indirecto en el rendimiento académico de los estudiantes en comprensión lectora.

Discusión y Conclusion: Los resultados obtenidos muestran que la aptitud intelectual es mejor predictor del desempeño en comprensión lectora, que las variables de apoyo familiar. Una posible razón es que, probablemente la subescala de Lengua y Literatura/Ciencias Sociales de SAGES-2, está altamente asociada con los contenidos curriculares relacionados con la comprensión de lectura. Sin embargo, es importante destacar el efecto de dos variables del contexto familiar. En primer lugar, el apoyo familiar autopercibido de los padres tuvo un efecto positivo, aunque con menor peso, en el desempeño del estudiante. El segundo aspecto es que el nivel de escolarización de los padres tuvo un efecto significativo en las aptitudes intelectuales evaluadas aquí.

Palabras clave: Apoyo, Padres, Rendimiento, Lectura, Aptitudes 


\begin{abstract}
Introduction. Students' academic performance can be influenced by various context and personal factors of the students. In the first case, for example, by the educational practice of their family of origin, and by the parental support with homework and learning at home. In the second case, for example, by the characteristics of the students, as well as by their intellectual aptitudes. The aim of this study was to obtain an explicative model of relationships between context variables (students' perception of family support, and parents's self-evaluation of support provided to their children), students' intellectual aptitudes, parents' educational level, and academic achievement in reading comprehension at the end of elementary school.
\end{abstract}

Method. The participants were 362 sixth graders from rural, indigenous and public schools in Sonora and Morelos, Mexico. The study was conducted during the academic course of 2015. Parents' support in learning their children at home was evaluated by mean of versions of the Family Support Perception Questionnaire (FSPQ), one for the parents and the other for students. The FSPQ evaluated four dimensions of support parents: 1. Homework Help /Reviewing, 2. Communication with school/Participation, 3. Conditions and Resources for studie in home, and 4. Monitoring/Communication with teachers. For assess the academic performance about Reading Comprehension was used an instrument attached to the curriculum for the sixth-grade of elementary school, with 17 items evaluating five dimensions: 1. Identifying spelling errors in five short texts, 2. Inferring implicit information and understand metaphors from narrative texts and short story, 3. Recognizing literary figures in poetry, 4. Inferring the meaning of words in the dominant indigenous language, and 5. Identifying sections of a newspaper. Intellectual aptitudes were measured using reduced versions of two scales of the Screening Assessment for Gifted Elementary and Middle School Students (SAGES-2), validated in Mexican context: 1. Thirteen items of the Language, Literature and Social Studies scale and 17 items for the General Reasoning scale.

Results. The results of a structural regression model with the EQS 6.4 program, showed that reading performance was explained mainly and significantly by the students' intellectual aptitudes, and secondly, by parent's support (evaluated by themselves). However, family support perceived by the students had no significant effects on reading performance. On the other hand, the parents' academic level showed a significant direct effect on students' intellectual abilities, and had as well an indirect effect on students' academic performance in reading comprehension.

\title{
Discussion and Conclusion.
}

The results show that the intellectual aptitude of the students is presented as a better predictor of their performance in reading comprehension than the variables of family support. One possible reason is that the Language Arts/Social Science subscale of SAGES-2 is probably highly associated with curricular content and learnings related to reading comprehension. However, it is important to highlight the effect of two variables in the family context. First, selfperceived parental support had a positive effect, although less so, on student performance. The second aspect is that the parents' level of schooling had a significant effect on the intellectual skills assessed here.

Keywords: Support, Parents, Performance, Reading, Aptitudes 


\section{Introducción}

El logro académico es la medida del nivel de aprendizaje alcanzado por un estudiante como resultado de un proceso complejo, y al mismo tiempo, como producto de la interacción entre numerosas variables. Las variables que influyen en el logro académico son diversas, y pueden estar relacionadas con la familia, la escuela, la comunidad, el entorno general, y con aspectos personales de los propios estudiantes (Kantor, 1975).

En el contexto de la familia, el apoyo que los padres brindan en casa en relación con las actividades escolares de sus hijos, sus expectativas e intereses, así como su participación en otras actividades promovidas por la escuela, han mostrado ser buenos predictores del rendimiento académico en los estudiantes (Fernández, Álvarez, Woitschach, Suárez, y Cuesta, 2017; Henderson y Mapp, 2002; LaRocque, Kleiman, y Darling, 2011; Núñez et al., 2015). Sin embargo, parecería que el apoyo de los padres de familia (ambos progenitores o adultos encargados del alumno) y su participación en las actividades escolares, son más frecuentes cuando la escuela proporciona programas eficientes para garantizar la participación de los padres.

Otra variable del entorno familiar que se ha relacionado con el aprendizaje y el logro académico es el nivel educativo de los padres, lo cual a su vez está altamente asociado con variables socioeconómicas. La literatura contemporánea sobre el tema muestra evidencias del efecto del nivel socioeconómico y educativo de las familias sobre los indicadores de aprendizaje y de rendimiento académico (Ho y Lam, 2016; Yang, 2017; Tan, Lyu, y Peng, 2019; Poon, 2020).

Estudios recientes en diversos contextos de habla hispana han demostrado el efecto del nivel educativo y cultural de la familia, sobre el logro académico, especialmente en evaluaciones a gran escala (Backhoff, 2011; Treviño, et al., 2010; Bazán, Backhoff, y Turullols, 2016). Del mismo modo, las evidencias confirman que los estudiantes que proceden de familias con más alto nivel cultural y socioeconómico - es decir, los que pueden contar con mejores oportunidades educacionales -, son los que tienen mayor posibilidad de obtener mejor nivel de rendimiento académico (Cueto, Guerrero, León, Zapata, y Freire, 2014; Cunha et al., 2015; Santos, Godás, Ferraces, y Lorenzo, 2016). 
Por otro lado, en lo que respecta a las variables de los estudiantes y su relación con el rendimiento académico, las aptitudes intelectuales en áreas específicas (por ejemplo, aptitudes linguísticas), también se ha considerado como variables que pueden proporcionar una mejor comprensión de las potencialidades de los estudiantes, y del uso que hacen de los recursos a los que tienen acceso en su aprendizaje escolar. Dado que la capacidad intelectual se ha examinado a menudo como una variable explicativa del rendimiento académico (Sternberg, 2008, 2010; Sternberg, Grigorenko, y Bundy, 2001), también ha sido ampliamente discutido en la literatura su papel como predictor del logro escolar.

En este sentido, estudios sobre la alta capacidad intelectual y las aptitudes sobresalientes, han examinado su relación con el logro académico, expresado en términos de promedio de rendimiento, o en términos de diferentes manifestaciones de aprendizaje (Ayoub y Aljughaiman, 2016; Cady, 2013; Gygy, Hagman-von Arx, Scheizer, y Grob, 2017; Lopes, Lemos, Primi, y Almeida, 2015). Sin embargo, algunos estudios han reportado que, las variables de contexto familiar, por ejemplo, el capital cultural de los padres, podrían influir en el apoyo educativo que brindan a sus hijos con alta capacidad (Castellanos, Bazán, Ferrari, y Hernández, 2015; Chávez y Acle, 2018; Grantham, et al., 2007; Kusumaningrum y Alsa, 2016; Karbach, Gottschling, Spengler, Hegewald, y Spinath, 2013; Phillipson y Phillipson, 2012; Rodríguez, Cadenas, Borges, y Valadez, 2019). Por otra parte, Karbach, Gottschling, Spengler, Hegewald y Spinath (2013) han señalado que, la participación de los padres en el progreso escolar de sus hijos es más importante que la capacidad cognitiva general, como predictor del rendimiento académico de los adolescentes en los ámbitos del lenguaje y las matemáticas, aunque no todos los tipos de participación familiar tienen el mismo impacto en el aprendizaje y el éxito académico de los estudiantes.

Diferentes estudios han respaldado la relación entre las variables de contexto, las aptitudes intelectuales y el logro académico. Sin embargo, relativamente poco se conoce sobre el efecto que en conjunto el nivel educativo de los padres, el apoyo familiar y las aptitudes intelectuales tienen sobre el logro escolar.

Por un lado, la mayoría de los estudios que analizan factores contextuales y personales, así como sus relaciones con el logro académico, han utilizado los resultados de las evaluaciones a gran escala como indicador de logro. Sólo algunos de estos estudios han incluido evaluaciones específicas de los resultados de aprendizaje asociados a los planes de estudios, e 
incluso han explorado las relaciones entre los diferentes indicadores de logro. Por otro lado, a pesar de la creciente importancia que la atención a los estudiantes con alta capacidad o aptitud intelectual sobresaliente ha adquirido en el contexto educativo mexicano (Valadez et al., 2016), falta profundizar en la relación entre esta y el desempeño académico.

\section{Objetivo e hipótesis}

El objetivo de este estudio fue identificar relaciones explicativas entre las variables de contexto de apoyo familiar para el estudio y el aprendizaje en el hogar, el nivel de aptitud intelectual de los estudiantes, y el desempeño en comprensión lectora, en sexto grado de primaria. Para identificar las relaciones entre las diferentes variables incluidas en este estudio, se construyó un modelo hipotético general de relaciones estructurales (véase la Figura 1). Una primera hipótesis del modelo supone que el desempeño en comprensión lectora es predicho significativamente y de manera simultanea, por el apoyo familiar en el estudio en casa (evaluado por medio de la autovaloración de los padres, y la percepción de los estudiantes), y por la aptitud intelectual del estudiante. Una segunda hipótesis es que el nivel educativo de los padres predice siginificativamente, tanto a la aptitud intelectual de sus hijos, como al apoyo que los padres les dan a sus hijos en el estudio en casa.

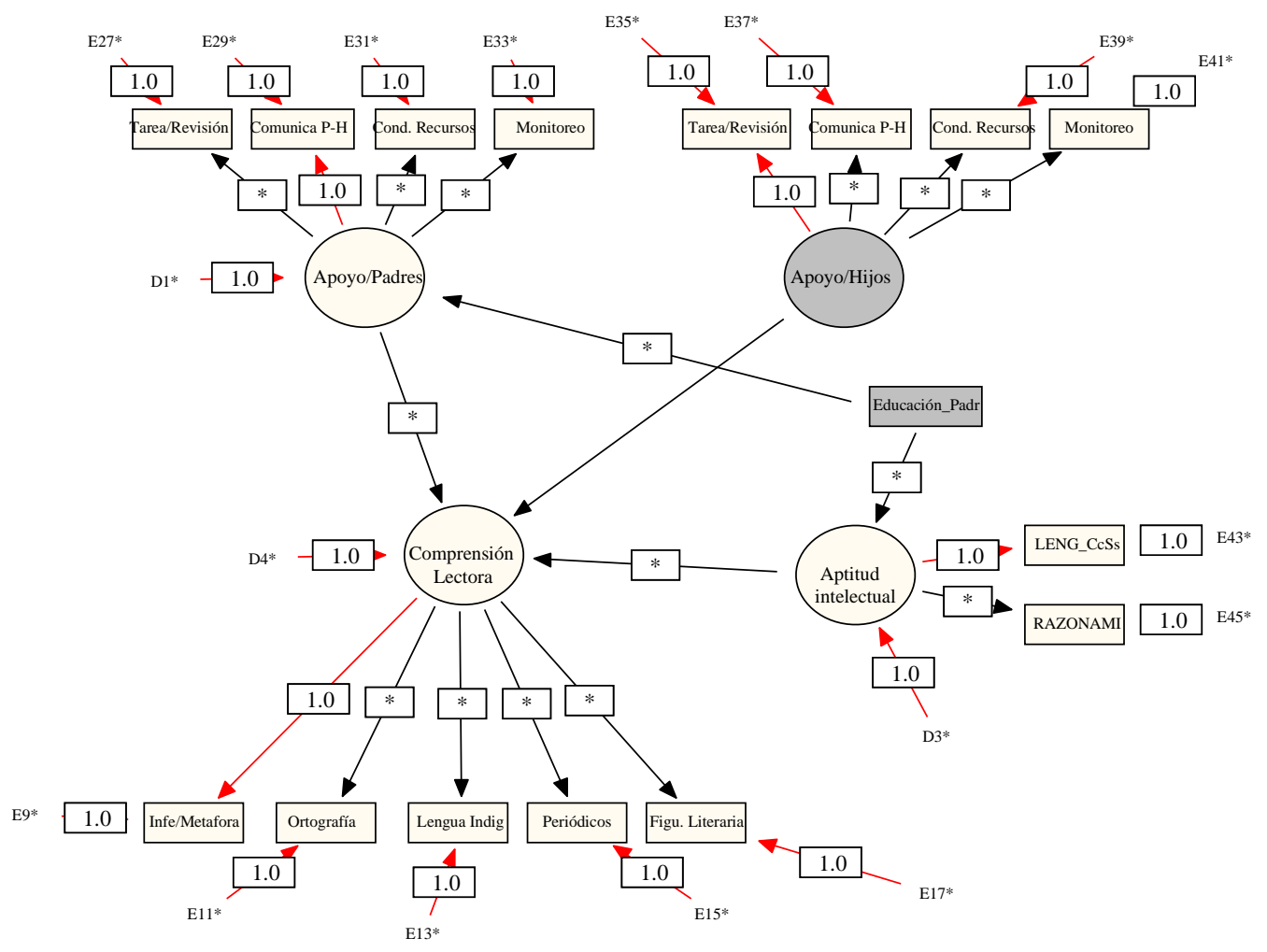

Figura 1. Modelo estructural hipotético de relaciones entre variables de apoyo de los padres, nivel educativo de padres, aptitud intelectual y el rendimiento en lectura 


\section{Método}

\section{Participantes}

En el presente estudio participaron 362 alumnos de sexto grado de primaria, con edades comprendidas entre los 11 y 14 años, provenientes de escuelas rurales - indígenas, privadas y públicas de Sonora y Morelos. Igualmente participaron sus padres.

\section{Instrumentos}

Cuestionario de Percepción de Apoyo Familiar (FSPQ). Este instrumento tiene dos versiones. Una de ellas es completada por los estudiantes, y evalúa la percepción del alumnado respecto al apoyo que les brinda sus padres en el aprendizaje escolar. La otra versión es completada por la madre o por el padre, respecto del apoyo que les brindan a sus hijos en su aprendizaje y estudios en casa. El cuestionario mostró indicadores de fiabilidad interna y externa adecuados, así como validez de constructo (Bazán y Castellanos, 2015). La aplicación tomó entre 15 y 25 minutos. Cada versión tiene 19 preguntas; las respuestas se dan en términos de frecuencia de la ocurrencia de los eventos, en una escala de 0 a 4 ( 0 - Nunca, 1 - Casi nunca, 2 - A veces, 3 - Casi siempre, 4 - Siempre). Una pregunta de las preguntas en ambas versiones, mide la cantidad de tiempo que los adultos dedican a ayudar a sus hijos en la tarea, en la asignatura Español, de acuerdo con la siguiente escala: $0=$ Menos de 30 minutos o Nunca, 1 = de 30 minutos a 1 hora, $2=$ de 1 hora a hora y media, $3 .=$ de hora y media a 2 horas; 4 $=2$ horas o más.

La versión $F S P Q$ para padres. Los datos relativos a la autoevaluación de los padres sobre el apoyo dado a sus hijos pasaron un análisis factorial exploratorio con EQS 6.2. Los resultados arrojaron 4 factores (prueba de KMO a 0,90; Prueba de Bartlett, X2 a 1938.980, p $=0.000$ ), que explicaron el $57 \%$ de la varianza total. Al mismo tiempo, todos los factores pasaron por un análisis factorial confirmatorio. Se especificaron cuatro constructos de Apoyo Familiar: 1. Ayuda en la tarea/Revisión (con 6 preguntas que exploran actividades de apoyo, revisión y comprobación de lecciones, y evaluaciones específicas del dominio); 2. Comunicación (con 5 preguntas relativas a la comunicación regular y a las conversaciones con los niños sobre su aprendizaje escolar, la tarea, las relaciones con los maestros y compañeros de clase, así como la participación en las reuniones escolares), 3. Condiciones y Recursos (con 4 preguntas relacionadas con las condiciones de las actividades de estudio, tales como disponibilidad de recursos y materiales educativos adicionales, tiempo y lugares asignados al estudio), y 
4. Monitoreo/Comunicación con los maestros (que abarca 2 preguntas sobre comunicación de los padres con los profesores para dar seguimiento al progreso y aprendizaje de sus hijos). El modelo confirmatorio resultante de la autoevaluación de los padres del apoyo prestado a sus hijos mostró validez convergente y divergente de constructo, y obtuvo una aceptable bondad de ajuste $(X 2=335.17, p=0.00, C F I=0.91, R M S E A=0.07)$.

La versión FSPQ para estudiantes. El análisis factorial exploratorio produjo cuatro factores que explicaron el $54 \%$ de la varianza (prueba de $K M O=0.91$, prueba de Bartlett: X2 $=1964.432, p=0.000)$. El análisis factorial confirmatorio con EQS 6.2 dió como resultado un modelo óptimo con validez divergente y convergente, con 4 constructos de percepción del apoyo familiar, y con una adecuada bondad de ajuste $(X 2=298.40, p=0.00, C F I=0.92$, RMSEA = 0,06): 1 . Ayuda en la tarea/Revisión (con 6 preguntas que exploran las actividades de apoyar la tarea, revisar lecciones y cuadernos, y ayudar a resolver las tareas escolares), 2. Comunicación Padres - Niños (con 3 preguntas que exploran la relación entre los estudiantes y sus padres, profesores, compañeros de escuela y la escuela), 3. Condiciones y recursos (5 preguntas relacionadas con las condiciones de las actividades de estudio, como la disponibilidad de recursos y materiales educativos adicionales, así como cuestiones de progreso y tareas escolares; 4. Seguimiento/Revisión (3 preguntas relativas a las actividades de los padres relativas a repasar, asignar ejercicios adicionales, evaluaciones y seguimiento del progreso de aprendizaje de sus hijos).

Nivel académico de los padres. Una pregunta relativa al nivel académico de los padres se añadió al cuestionario FSPQ para los padres (grado o nivel escolar del padre o la madre).

Comprensión lectora. Para evaluar el desempeño académico en lectura se utilizó un instrumento que corresponde al currículo oficial establecido por la Secretaría de Educación pública en México (SEP) para el sexto grado de la escuela primaria. Este instrumento tiene 17 elementos (tareas); se organizó en base al programa curricular de dominio de la Lengua Española para el sexto grado (nivel educativo elemental en México), y abarca cinco bloques o áreas de aprendizaje, cada uno de ellos correspondiente a su vez a un bloque bimensual. Cada bloque del programa abarca diferentes actividades con respecto a la comprensión de lectura.

Al mismo tiempo, estas actividades de evaluación se construyeron de acuerdo con los objetivos de aprendizaje esperados que el programa oficial pretende alcanzar. Completar las 
preguntas del instrumento toma de 40 a 45 minutos. Las respuestas se evalúan con valores que van de 1 a 4 en una escala inclusiva de logro, según el cumplimiento de criterios cualitativamente jerarquizados de menor a mayor complejidad. No se consideran respuestas incorrectas, sólo diferentes niveles de logro en cada pregunta. El estudiante puede alcanzar un valor máximo de 4 en cada pregunta si su respuesta cumple con todos los criterios de logro, establecidos en una hoja de rúbricas, con ayuda de la cual cada cuestionario fue calificado.

Como primer paso, se llevó a cabo un análisis factorial exploratorio, resultando cinco factores que en conjunto explicaron el $71 \%$ de la varianza, con una medida adecuada de muestreo (medida Kaiser-Meyer-Olkin = 0.74), utilizando el método de extracción ortogonal, rotación Varimax con la normalización de Kaiser en 5 iteraciones.

Los cinco factores fueron: 1. Identificación de errores ortográficos en textos cortos (en total cinco elementos), con un índice de consistencia interna de .87 (coeficiente alfa de Cronbach); 2. Inferir información implícita y metáforas en textos narrativos (dos temas sobre un acontecimiento histórico y otros dos, de un cuento sobre un joven que viajó lejos para saber qué era el miedo), con un índice de consistencia interna de .67; 3. Reconociendo figuras literarias en la poesía (dos preguntas de la lectura de poemas cortos), que obtuvo un coeficiente alfa de Cronbach $=.87 ; 4$. Inferir el significado de palabras en la lengua indígena dominante de la región donde viven los participantes, obtuvo un índice con consistencia de .85 (dos artículos en un cuento corto en Náhuatl para los estudiantes del Estado de Morelos, y dos artículos en un cuento corto en Yaqui para los alumnos de Sonora), y 5. Identificación de secciones del periódico (dos temas independientes de textos en desorden en diferentes secciones de un periódico), con un coeficiente alfa de Cronbach $=.84$.

Una vez obtenida la estructura factorial explorada sobre comprensión lectora en Español 6to grado, se llevó a cabo un análisis factorial confirmatorio, utilizando el programa EQS 6.2; resultando buena validez convergente y divergente de constructo. El modelo confirmatorio obtuvo acceptable bondad de ajuste respecto al modelo hipotético basado en los cinco factores y sus variables manifiestas (elementos), derivados del análisis factorial exploratorio $(P=$ .00; Indice de Ajuste Comparativo CFI = .93; El índice de ajuste de Bollen IFI = .94; RMSEA $=.07)$. 
Evaluación de aptitudes intelectuales. Para el presente estudio se utilizaron las versiones reducidas de dos escalas (Lengua y Literatura/Ciencias Sociales, y Razonamiento General), de las tres escalas que conforman la prueba original Screening Assessment for Gifted Elementary and Middle School Students (SAGES) Nivel 2, de Johnsen y Corn (2003). La SAGES-2 (en español, Evaluación Inicial para Estudiantes con Aptitudes Sobresalientes) es una prueba colectiva utilizada para identificar a niños de 5 a 14 años de edad con aptitudes intelectuales en los ámbitos académicos y de razonamiento: Matemáticas / Ciencias Naturales (30 ítems), Lengua y Literatura/Ciencias Sociales (30 ítems), y Razonamiento General (35 ítems).

La SAGES, en su versión para escolares de cuarto grado de primaria a segundo año de secundaria, fue adaptado y utilizado en México por Sánchez y Acle en 2003 (Valdés y Vera, 2012). El instrumento contiene elementos de formato de opción múltiple.

\section{Escala Lengua y Literatura/Ciencias Sociales.}

Para el presente estudio fueron tomados 16 preguntas de la subescala 2 (Lengua y Literatura/Ciencias Sociales, que en un análisis factorial exploratorio conformaron un primer factor, la cual explicó el $17 \%$ de la varianza total. El análisis factorial confirmatorio con EQS 6.2 para determinar la dimensionalidad de esta escala, confirmó un factor llamado Aptitudes en Lenguaje y Ciencias Sociales, con 13 indicadores (elementos). Asimismo, el modelo resultante obtuvo aceptables indicadores prácticos de Bondad de Ajuste (Chi Square $=98.68, p=$ $0.00, C F I=0.90, R M S E A=.04)$.

\section{Escala de Razonamiento general.}

Se seleccionó el primer factor resultante del análisis factorial exploratorio realizado en la subescala Razonamiento general, con 17 elementos, que explica el 54\% de la varianza. Mediante un análisis factorial confirmatorio, se obtuvo un modelo de 17 elementos (indicadores), con una aceptable bondad de ajuste ( $C h i S q .=296.52, P=0.00, C F I=0.90, R M S E A=0.07)$.

\section{Consideraciones éticas}

Este estudio se llevó a cabo de acuerdo con las recomendaciones de los Principios Eticos de los Psicólogos y el Código de Conducta de la Asociación Americana de Psicología (APA, 2017). El protocolo de investigación (No 15981-2010) fue aprobado por el Consejo Nacional de Ciencia y Tecnología (CONACYT) de México, dentro de la Convocatoria de 
Ciencias Básicas para Proyectos de Investigación 2010. El protocolo de investigación obtuvo la aprobación de los revisores pares de CONACYT, que incluye, entre todos los parámetros de su evaluación, las consideraciones éticas y las declaraciones de acuerdo con los principios del Código Mexicano del Psicólogo y la APA. Por lo tanto, en el momento en que se llevó a cabo la investigación, CONACYT era el organismo responsable de proporcionar la aprobación ética.

El procedimiento de consentimiento incluyó una reunión introductoria con los padres de familia y el director de la escuela con el fin de explicar el proyecto y exponer oralmente diferentes cuestiones relacionadas con las condiciones y requisitos éticos, tales como: los objetivos del proyecto, el carácter voluntario de la participación (como la posibilidad de retirarse), el propósito científico y el uso confidencial de los resultados de la investigación, la confidencialidad (anonimato) de los nombres de las personas y escuelas, y en general, de los datos obtenidos.

La aplicación de los instrumentos utilizados en la investigación (los cuestionarios para los padres y para los estudiantes, la prueba de aptitudes, así como el instrumento para la evaluación de las competencias académicas de los escolares) requirieron un consentimiento informado de acuerdo con la Declaración de Helsinki de la Asociación Médica Mundial. Los padres de familia (o tutores legales) firmaron un consentimiento informado para autorizar la participación de sus hijos/as o tutorado/da. Asimismo, el director o la directora de cada escuela particpante, completó un formato escrito de asentimiento para el desarrollo de las aplicaciones en dichas escuelas.

Para desarrollar esta investigación, se obtuvo autorización por escrito de parte del Instituto de Educación Básica de Morelos (IEBEM), lo cual se informaba al director o a la directora de cada escuela. Además, en la presentación y la discusión de resultados, se observaron igualmente las normas éticas de confidencialidad con respecto a los nombres de los participantes y de las propias escuelas.

Por último, no se manfiestaron conflictos de intereses por parte de los investigadores relativos a la realización o publicación de los resultados de la investigación. 


\section{Procedimiento}

Una vez obtenido el permiso de las autoridades educativas, se celebraron reuniones con los padres de familia o tutores legales, y después con los alumnos, en las que se explicaron los objetivos del estudio y sus implicaciones éticas de la participación voluntaria. Luego se proporcionó un resumen de los objetivos del estudio con un formulario para su consentimiento informado a los padres y estudiantes.

Más adelante, se aplicó el cuestionario de apoyo familiar a los padres de familia. Los que no asistieron a la reunión, recibieron una carta de la escuela con información sobre los objetivos del proyecto, y también, adjunto en un sobre, el formulario de consentimiento informado, y el cuestionario sobre el apoyo de los padres.

Las competencias de lectura de los estudiantes y la percepción del apoyo familiar se evaluaron en dos sesiones, mientras que en una tercera sesión se aplicó la prueba SAGES 2. Las autoridades escolares determinaron un calendario para las sesiones de evaluación que se llevaron a cabo en las propias aulas de los estudiantes con el apoyo de los maestros de grupo. Además, tres estudiantes del último año de licenciatura en psicología llevaron a cabo la administración de los instrumentos de evaluación; anteriormente fueron entrenados con una guía básica para la evaluación, las cartas descriptivas y los formatos de registro.

\section{Análisis de datos}

Utilizando el paquete estadístico SPSS 23, se realizó un análisis descriptivo de las variables incluidas en el estudio. Además, utilizando el programa estadístico EQS 6.2, se sometió a prueba el modelo hipotético de regresión estructural que se mostró en la figura 1.

La variable latente Comprensión lectora fue considerada como la variable a predecir en el modelo de la figura 1. Esta variable latente fue conformada por cinco variables manifiestas que corresponden a los cinco componentes resultantes del análisis factorial exploratorio realizado anteriormente: 1. Infererencia/Metáfora (Inferir información implícita en textos narrativos y entender metáforas), 2. Ortografía (Identificar errores ortográficos en textos), 3. Lengua indígena (Inferir el significado de las palabras en la lengua indígena dominante; Náhuatl en Morelos y Yaqui en Sonora), 4. Periódico (Identificar secciones en un periódico), y 5. Figuras literarias (Reconocer figuras literarias en la poesía, así como su función de evocar emociones). 
La variable latente (o factor) aptitud intelectual fue construida a partir de la proporción de respuestas, sobre la suma total de cada una de las dos subescalas adaptadas de SAGES-2 (versión mexicana). LENG_CsSs es la proporción de respuestas correctas en la subescala Lengua y Literatura/Ciencias Sociales, considerando el 100\% del éxito cuando el estudiante responde con 1 a los 13 elementos. RAZONAMI: Es la proporción de respuestas correctas en la subescala de Razonamiento General. Se alcanza el 100\% si el alumno responde correctamente a los 17 elementos.

El nivel educativo de los padres refiere al nivel de escolaridad de los progenitores y / o adultos responsables de los estudiantes. Según el modelo hipotético, esta variable predice positivamente, tanto el apoyo proporcionado por los padres (percibido por éstos y por sus hijos), como las aptitudes intelectuales de los niños.

La escala de autovaloración de los padres, sobre el apoyo que les brindan a sus hijos con las tareas y el estudio en casa (Apoyo/Padres) comprendió cuatro indicadores, que son los índices de cada uno de los cuatro factores resultantes del Análisis Factorial Exploratorio (EFA) y del Análisis de Factorial Confirmatorio (AFC): Ayuda en la Tarea/Revisión, Comunicación Padres-Niños, Condiciones y Recursos, y Monitoreo.

El factor Apoyo Familiar percibido por el alumnado (Apoyo/Hijos) comprendió la estimación del estudiante sobre el apoyo recibido de sus padres o tutores, en cuatro dimensiones, también resultante de los AFE y AFC: Ayuda en la Tarea/ Revisión, Comunicación Padres-Niños, Condiciones y Recursos, y Monitoreo.

\section{Resultados}

\section{Análisis descriptivos}

En la Tabla 1 se presentan los resultados de los análisis descriptivos de cada uno de los factores del instrumento de comprensión de lectura, y las dos subescalas de la SAGES-2 utilizadas en el estudio. En cada caso, el valor total de la subescala corresponde al $100 \%$ de las respuestas posibles o correctas. Como se puede notar, con respecto a la comprensión lectora, las mejores puntuaciones de los estudiantes fueron en inferir el significado de las palabras 
usando su lengua materna (Náhuatl o Yaqui), así como, el reconocimiento de poesías, su expresión emocional y su función.

Por otro lado, los resultados del instrumento de apoyo familiar (Tabla 1), muestran que los padres tienen una mejor apreciación de su comunicación con sus hijos. Del mismo modo, la revisión y el monitoreo de la tarea de los niños tuvo una mejor evaluación en contraste con proporcionar condiciones y recursos materiales para el estudio y aprendizaje escolar en casa.

Tabla 1. Descripción de las variables inlcuidas en el estudio

\begin{tabular}{|c|c|c|c|c|c|c|}
\hline \multirow[b]{2}{*}{ Variables } & \multirow[b]{2}{*}{$N$} & \multirow[b]{2}{*}{ Rango } & \multicolumn{2}{|c|}{ Puntajes } & \multirow[b]{2}{*}{ Mean } & \multirow[b]{2}{*}{$S D$} \\
\hline & & & Min. & Max. & & \\
\hline \multicolumn{7}{|c|}{ Proporción de logro en cada factor de comprensión de lectura } \\
\hline Inferencia / Metáforas $(16=100 \%)$ & & & & & & \\
\hline $\begin{array}{l}\text { Inferir información implícita y entender me- } \\
\text { táforas en textos narrativos o historias. } \\
\text { Ortografía }(\mathbf{2 0}=\mathbf{1 0 0 \%})\end{array}$ & 347 & 100.00 & .00 & 100 & 22.42 & 20.67 \\
\hline $\begin{array}{l}\text { Identificación de errores ortográficos en } \\
\text { textos. }\end{array}$ & 347 & 100.00 & .00 & 100 & 32.81 & 35.02 \\
\hline $\begin{array}{l}\text { Lengua Indígena }(\mathbf{8}=\mathbf{1 0 0 \%}) \\
\text { Inferir el significado de palabras en la lengua } \\
\text { indígena náhuatl (Morelos) o Yaqui (Sono- } \\
\text { ra). }\end{array}$ & 347 & 100.00 & .00 & 100 & 41.17 & 35.26 \\
\hline $\begin{array}{l}\text { Periódico }(\mathbf{8}=\mathbf{1 0 0 \%}) \\
\text { Identificación de secciones de un periódico. } \\
\text { Figuras literarias }(8=\mathbf{1 0 0 \%})\end{array}$ & 347 & 100.00 & .00 & 100 & 24.86 & 26.60 \\
\hline $\begin{array}{l}\text { Reconocer figuras literarias en la poesía y su } \\
\text { función emocional. }\end{array}$ & 347 & 62.50 & .00 & 62.50 & 3.42 & 9.95 \\
\hline
\end{tabular}

$\overline{\text { Proporción de respuestas en cada dimensión de autoevaluación de apoyo familiar (Padres) }}$

Ayudar con tareas / Revisión (32 = 100\%)

Proporcionar ayuda con las tareas para la 359

100.00

$.00 \quad 100.00 \quad 46.89$

22.80

casa, y revisar sus cuadernos.

Comunicación Padres-Niños $(20=100 \%)$

Mantener comunicación con sus hijos sobre su aprendizaje en el salón de clases, la tarea

$\begin{array}{llllll}356 & 100.00 \quad .00 & 100.00 & 76.88 & 21.80\end{array}$

y su comportamiento escolar.

Condiciones y Recurses $(16=100 \%)$

Proporcionar condiciones de estudio en el hogar, disponibilidad de recursos y materia$\begin{array}{llllll}357 & 100.00 & .00 & 100.00 & 70.01 & 22.67\end{array}$ les educativos a sus hijos.

Monitoreo / Comunicación con profesores $(8=100 \%)$

Mantener comunicación con los maestros para dar seguimiento al progreso de aprendizaje de sus hijos. 
Tabla 1 (continua). Descripción de las variables inlcuidas en el estudio

\begin{tabular}{|c|c|c|c|c|c|c|}
\hline \multirow[b]{2}{*}{ Variables } & \multirow[b]{2}{*}{$\mathbf{N}$} & \multirow[b]{2}{*}{ Rango } & \multicolumn{2}{|c|}{ Puntajes } & \multirow[b]{2}{*}{ Mean } & \multirow[b]{2}{*}{ SD } \\
\hline & & & Min. & Max. & & \\
\hline \multicolumn{7}{|c|}{$\begin{array}{l}\text { Proporción de respuestas en cada dimensión de evaluación del Apoyo Familiar (Estudian- } \\
\text { tes) }\end{array}$} \\
\hline Ayuda con tarea / Revisión $(24=100 \%)$ & & & & & & \\
\hline $\begin{array}{l}\text { Los padres ayudan a los estudiantes con sus } \\
\text { tareas, revisa cuadernos y resuelven ejerci- } \\
\text { cios juntos. }\end{array}$ & 347 & 100.00 & .00 & 100.00 & 50.03 & 23.82 \\
\hline $\begin{array}{l}\text { Comunicación Padres-Niños }(\mathbf{1 2}=\mathbf{1 0 0 \%}) \\
\text { Los padres preguntan sobre su relación con } \\
\text { los maestros, compañeros de clase y la escue- } \\
\text { la en general. }\end{array}$ & 348 & 100.00 & .00 & 100.00 & 81.06 & 22.60 \\
\hline $\begin{array}{l}\text { Condiciones and Recursos }(\mathbf{2 0}=\mathbf{1 0 0 \%}) \\
\text { Los padres proporcionan condiciones de } \\
\text { estudio en el hogar, disponibilidad de recur- } \\
\text { sos y materiales educativos a sus hijos. }\end{array}$ & 347 & 100.00 & .00 & 100.00 & 71.45 & 22.26 \\
\hline $\begin{array}{l}\quad \text { Monitoreo / Revisión }(\mathbf{1 2}=\mathbf{1 0 0 \%}) \\
\text { Los padres realizan actividades de ensayo, y } \\
\text { asignan ejercicios adicionales, evaluaciones } \\
\text { y seguimiento del progreso en aprendizaje de } \\
\text { sus hijos. }\end{array}$ & 347 & 100.00 & .00 & 100.00 & 47.21 & 26.11 \\
\hline \multicolumn{7}{|c|}{ Proporción de respuestas en las dos subescalas of aptitude intelectual } \\
\hline $\begin{array}{l}\text { Lengua, Literatura y Ciencias Sociales, } 13 \\
\text { items }(13=100 \%)\end{array}$ & 325 & 92.31 & .00 & 92.31 & 41.91 & 20.51 \\
\hline $\begin{array}{l}\text { Razonamiento general, } 17 \text { ítems }(17= \\
100 \%)\end{array}$ & 322 & 100.00 & .00 & 100.00 & 47.26 & 28.73 \\
\hline
\end{tabular}

La Tabla 1 muestra que los estudiantes perciben como más frecuente, la comunicación padres- hijo, coincidiendo con la apreciación de sus padres. Sin embargo, en contraposición con sus padres, valoran como más frecuentes las actividades que éstos desarrollan para garantizar y poner a su disposición recursos y condiciones en el hogar que garanticen el estudio y las tareas escolares.

La Tabla 2 muestra el nivel de educación de los padres. Menos de la mitad de los padres $(36,8 \%)$ tienen estudios en el nivel superior (técnico o universitario) y post universitario. 
Tabla 2. Nivel educativo del padre o madre (o tutor) quien respondió el cuestionario de autoevaluación de apoyo familiar

\begin{tabular}{lcccc} 
Nivel educativo & Frecuencia & Porcentaje & $\begin{array}{c}\text { Porcentaje } \\
\text { válido }\end{array}$ & $\begin{array}{c}\text { Porcentaje } \\
\text { acumulado }\end{array}$ \\
\hline Educación primaria & 99 & 27.3 & 30.4 & 30.4 \\
Educación secundaria & 107 & 29.6 & 32.8 & 63.2 \\
Educación media & 55 & 15.2 & 16.9 & 80.1 \\
Estudios de educación & 65 & 18.0 & 19.9 & 100.0 \\
superior o Posgrado & 326 & 90.1 & 100.0 & \\
Total & 36 & 9.9 & & \\
perdidos & $\mathbf{3 6 2}$ & $\mathbf{1 0 0 . 0}$ & & \\
\multicolumn{1}{c}{ Total } & & &
\end{tabular}

Relación entre las variables asociadas y el logro

La Figura 2 muestra el modelo resultante para el rendimiento en comprensión lectora en estudiantes de sexto grado, que obtuvo una aceptable bondad de ajuste (CFI $=0.98$, RMSEA = .09) y que, por lo tanto, se puede considerar como similar al modelo hipotético que fue presentado en la Figura 1, conocido también como un modelo esperado o teorizado. Sin embargo, en el modelo resultante que se muestra en la Figura 2, fue necesario eliminar la variable Figura literaria, porque explicó sólo el 2\% de la varianza obtenida. Del mismo modo, debido a la parsimonia del modelo, se eliminó la variable MonitoreP (Monitoreo), ya que su inclusión afectaba a la bondad de ajuste del modelo.

El modelo obtenido permite señalar que el rendimiento en lectura, conformado por cuatro índices de dominio de la comprensión lectora en sexto grado de primaria, fue explicado de manera significativa, principalmente por el factor aptitud intelectual del estudiante y en menor medida, por el factor apoyo de los padres cuando este es auto-valorado por los propios padres (padre o madre). A su vez, el factor aptitud intelectual fue significativamente afectado por el nivel educativo de los padres. Es decir, el nivel educativo de los padres tuvo una influencia indirecta, en el desempeño de los estudiantes en comprensión lectora, mediada por la aptitud intelectual.

No se confirmaron las relaciones predictivas entre el apoyo de los padres percibido por los niños y el desempeño de los alumnos en comprensión lectora; tampoco se confirmó el efecto del nivel educativo de los padres sobre su autovaloración del apoyo brindado a sus hijos. Por el contrario, el programa sugirió establecer en el modelo, una co-varianza entre apoyo 
familiar autovalorado por los padres y el apoyo familiar percibido por los hijos, y esta covarianza fue alta y significativa (0.95).

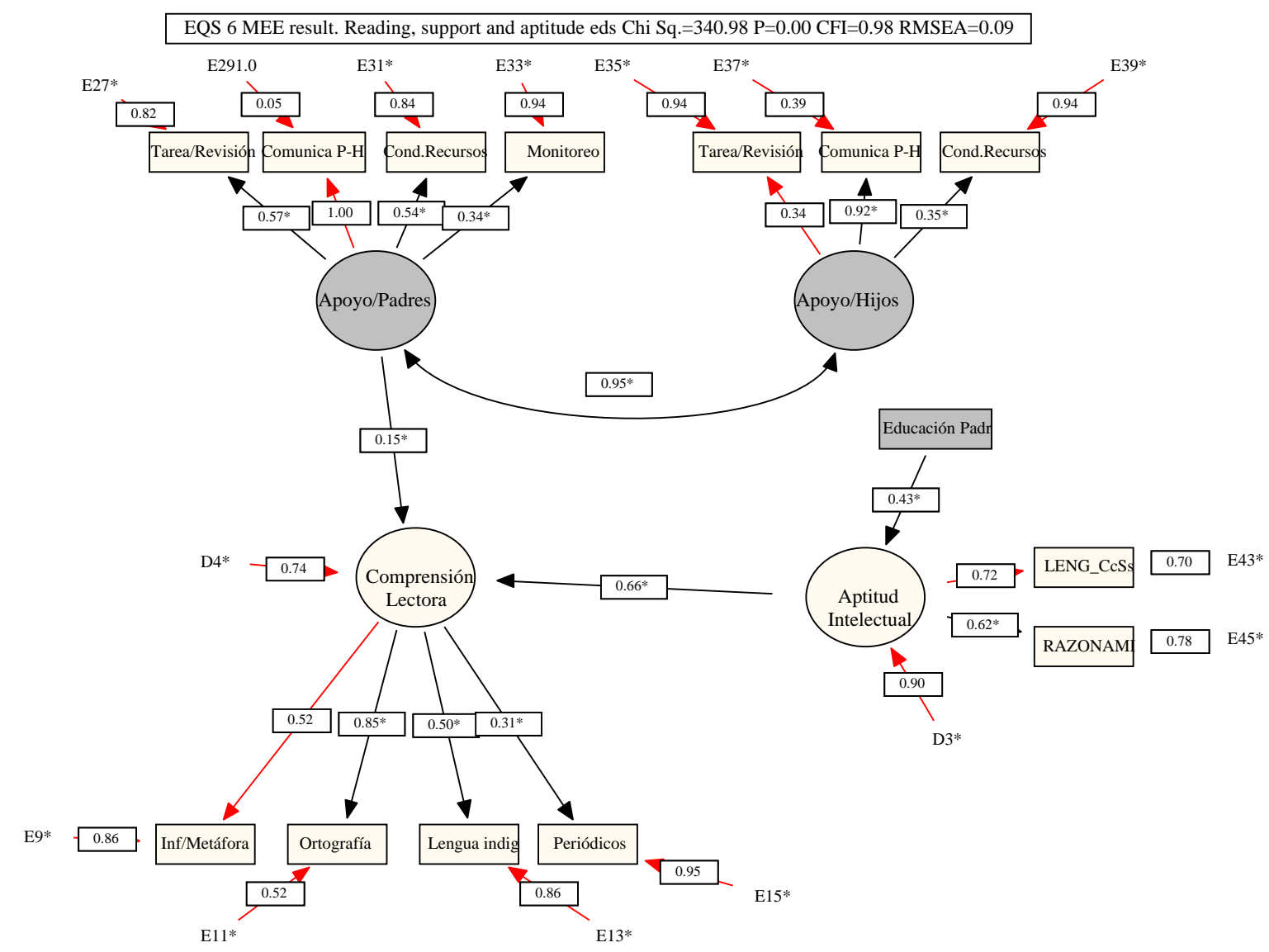

Figura 2. Modelo estructural resultante, de relaciones entre variables de apoyo de los padres, nivel educativo de padres, aptitud intelectual y rendimiento en lectura

\section{Discusión}

Un primer aspecto a tener en cuenta en este estudio es que el rendimiento en comprensión lectora fue explicado signficativamente por las aptitudes intelectuales de los estudiantes (mostradas en el área verbal y en el de razonamiento). Las evidencias coinciden con hallazgos de otras investigaciones que han señalado la influencia de variables relacionadas con la inteligencia y altas habilidades en el logro académico (Ayoub y Aljughaiman, 2016; Cady, 2013; Gygy et al., 2017; Lopes et al., 2015). 
De hecho, Sternberg y otros (2001) han discutido sobre las posibles causas de estas relaciones, haciendo hincapié en los nexos existentes entre los procesos y las habilidades necesarias para resolver las tareas académicas, las implícitas en la evaluación de la inteligencia y las habilidades y destrezas necesarias para el aprendizaje escolar. Por lo tanto, resulta comprensible que los resultados del presente estudio señalen que las habilidades intelectuales que forman parte de las competencias de comprensión lectora (rendimiento en clases de español en el currículo mexicano), también están presentes en las tareas de la subescala 2 de SAGES2 (Johnsen y Corn, 2003). Además, la SAGES 2 evalúa aspectos generales de la capacidad de razonamiento lingüístico, lógico y abstracto, como lo planteó Martínez-Otero (2002).

Un segundo punto a resaltar es el hecho de que la aptitud intelectual tenga un mayor efecto sobre el logro académico, comparado con el efecto de la variable apoyo familiar autovalorada por los padres de familia. Una posible razón para que la aptitud intelectual tenga más peso explicativo en el logro, en comparación con el efecto del apoyo de los padres, podría deberse a un contenido curricular fuerte o saturado en la subescala Lengua y Literatura/Ciecias Sociales de la SAGES 2, y que esta alta asociación se deba a las similitudes entre los contenidos y tareas que se enseñan en las aulas y la evaluación sobre la lectura, con esta versión de la SAGES. Hallazgos y explicaciones similares fueron informados por Castellanos et al. (2015).

Adicionalmente, los resultados del presente estudio corroboran el análisis realizado por Sternberg $(2001,2008,2010)$ y por otros expertos en la evaluación de la capacidad intelectual, como Santamaría, Arribas, Pereña y Seisdedos (2005), en los que se destaca que la aptitud intelectual, más que una capacidad homogénea, integra un amplio diapasón de habilidades y procesos, que deben expresarse tanto en la subprueba de razonamiento, como en las exigencias de las tareas linguísticas (Johnsen y Corn, 2003), y en términos de capacidades de pensamiento lógico-abstracto y analógico.

Como tercer punto a destacar, los resultados de esta investigación confirman que el nivel académico de los padres está fuertemente asociado con los indicadores de la capacidad o aptitud intelectual del alumno. Tomando en cuenta que el factor aptitud intelectual fue predicho significativamente por la variable nivel educativo de los padres y que, a la vez, el factor aptitud intelectual tuvo un efecto significativo sobre el logro académico, es posible suponer 
que esta relación aptitud intelectual - logro, puede depender del nivel académico de los padres y del capital cultural de las familias de origen.

Varios estudios con familias de alumnos mexicanos con alta capacidad intelectual y académica, han mostrado que, el nivel educativo de los progenitores y el capital cultural de la familia, están asociados con las prácticas y las actividades educativas que los padres realizan con sus hijos en casa para potenciar sus habilidades y capacidades, así como con el apoyo en las tareas escolares y el estudio en el hogar (Bazán, Navarro, Treviño, y Castellanos, 2014; Castellanos, et al., 2015; Chávez y Acle, 2018). Un mayor capital educativo y cultural del entorno familiar, así como las prácticas sistemáticas de educación y apoyo de los padres en las tareas escolares, tendrían un efecto positivo en el logro académico (Altschul, 2012; Grantham, et al., 2007; Kusumaningrum y Alsa, 2016; Phillipson y Phillipson, 2012).

Un cuarto aspecto a tener en cuenta es el efecto diferencial del apoyo parental sobre el rendimiento en comprensión lectora, dependiendo de la autoevaluación de los propios padres de familia, o la percepción de los estudiantes. Se encontró una relación significativa entre el apoyo familiar cuando fue reportado por los padres, y el rendimiento en lectura, pero no se encontró ninguna relación significativa entre el apoyo de los padres percibido por los estudiantes y su desempeño en la lectura.

Los resultados de este estudio mostraron que el apoyo de los padres tiene efectos positivos en los indicadores de logro académico, y coinciden con los hallazgos de otros investigadores, por ejemplo, LaRocque, Kleiman y Darling (2011), Karbach et al. (2013). Los resultados de este estudio también apoyan la importancia de reunir información sobre el apoyo familiar, de los propios padres, como se hace en los cuestionarios de evaluaciones a gran escala (OCDE, 2014). La autoevaluación de los padres ha demostrado tener un efecto positivo y significativo en el desempeño de los estudiantes mexicanos, tanto en evaluaciones a gran escala como también, en evaluaciones a pequeña escala (Bazán y Castellanos, 2015; Bazán et al., 2016).

Por otro lado, el factor apoyo familiar académico percibido por el estudiante, no tuvo efecto en el factor rendimiento en comprensión lectora, pero en el modelo resultante, se encontró una co-varianza significativa con el apoyo familiar autocalificado por uno de sus progenitores (padre o madre). Al parecer, el apoyo de los padres para el aprendizaje en casa, per- 
cibido por el estudiante, tiene un efecto importante sobre el rendimiento académico, pero asociado con el compromiso académico del alumno y un mayor tiempo y esfuerzo dedicados a la realización de sus tareas (Chen, 2005; Núñez, et al., 2015).

Estos hallazgos apuntan hacia la necesidad de seguir investigando sobre la relación entre el nivel educativo de los padres, las aptitudes intelectuales, el apoyo de los padres en el aprendizaje en casa y el logro académico. Es importante seguir contribuyendo a la investigación de variables familiars, los cuáles tienen una influencia directa o indirecta sobre el rendimiento académico de los estudiantes, ya que las diferencias entre los estudiantes con respecto al logro académico podrían estar indicando que las familias de origen tienen una gran influencia en el desarrollo de la lectura (Fernández, et al., 2017; Treviño et al., 2010).

\section{Declaración de financiamiento}

Consejo Nacional de Ciencia y Tecnología de México, CONACYT. Proyecto de Investigación No 151981, 2012 - 2016.

\section{Referencias}

Altschul, I. (2012). Linking socioeconomic status to the academic achievement of Mexican American youth through parent involvement in education. Journal of the Society for Social Work and Research, 3(1), 13-30. doi: 10.5243/jsswr.2012.2

Ayoub, A. E. A., y Aljughaiman, A. M. (2016). A predictive structural model for gifted students' performance: A study based on intelligence and its implicit theories. Learning and Individual Differences, 51, 11-18. https://doi.org/10.1016/j.lindif.2016.08.018

Backhoff, E. (2011). La inequidad educativa en México: diferencias en el aprendizaje de la comprensión lectora en educación básica. Profesorado, Revista de Currículum y Formación del Profesorado, 15(3), 87-102.

Bazán, A., y Castellanos, D. (2015). Parents' Support and Achievement in a Mexican Elementary School: Child's Perception and Parents' Self-Evaluation. International Journal of Psychological Studies, 7(4), 59-69. doi: 10.5539/ijps.v7n4p59

Bazán, A., Backhoff, E., y Turullols, R. (2016). School participation, family support, performance in mathematics: The case of Mexico in PISA (2012). RELIEVE, Revista Electrónica de Investigación y Evaluación Educativa, 22(1), art. M5. https://ojs.uv.es/index.php/RELIEVE/article/view/8242/11938 
Bazán, A., Navarro, A.L., Treviño, S., y Castellanos, D. (2014). Estrategias de apoyo de padres de alumnos de alto desempeño, para el estudio y aprendizaje escolar. Profesorado, Revista de Currículum y Formación del Profesorado,18(2), 153-175. https://ojs.uv.es/index.php/RELIEVE/article/view/8242/11938

Cady, J. A. (2013). The implications of a high academic ability learning environment on third grade gifted students'academic achievement Florida public schools (Doctoral dissertation), University of Central Florida Orlando, Florida.

Castellanos, D., Bazán, A., Ferrari, A., y Hernández, C. (2015). Apoyo familiar en escolares de alta capacidad intelectual de diferentes contextos socioeducativos. Revista de Psicología, 3(2), 299-332.

Chávez, B., y Acle, G. (2018). Children with High capacities: Analyses of the relatives' variables involved in the development of the potential. Electronic Journal of Research in Educational Psychology, 16(2), 273-300.

Chen, J. (2005). Relation of academic support from parents, teachers, and peers to Hong Kong adolescents' academic achievement: The mediating role of academic engagement. Genetic, Social, and General Psychology Monographs, 131(2), 77-127. doi: 10.3200/MONO.131.2.77-127

Cueto, S., Guerrero, G., León, J., Zapata, M., y Freire, S. (2014). The relationship between socioeconomic status at age one, opportunities to learn and achievement in mathematics in fourth grade in Peru. Oxford Review of Education, 40(1), 50-72. doi: $10.1080 / 03054985.2013 .873525$

Cunha, J., Rosário, P., Macedo, L., Nunes, AR., Fuentes, S., Pinto, R., y Suárez, N. (2015). Parents' conceptions of their homework involvement in elementary school. Psicothe$m a, 27$ (2) 159-165. doi: 10.7334/psicothema2014.210

Fernández, R., Álvarez, M., Woitschach, P., Suárez, J., y Cuesta, M. (2017). Parental involvement and academic performance: Less control and more communication. Psicothema, 29(4), 453-461. doi: 10.7334/psicothema2017.181

García, O., López, F., Icaran, E., Burgos, S. (2014). Relationship between general intelligence, competences and academic achievement among university students. Personality and Individual Differences, 60, S67. https://doi.org/10.1016/j.paid.2013.07.296

Grantham-McGregor, S., Cheung, Y. B., Cueto, S., Glewwe, P., Richter, L., Strupp, B., y International Child Development Steering Group (2007). Developmental potential in the first 5 years for children in developing countries. The Lancet, 369, 60-70. 
Gygy, J. T.; Hagman-von Arx, P.; Scheizer, F.; y Grob, A. (2017). The Predictive Validity of Four Intelligence Tests of School Grades: a small sample longitudinal study. Frontiers in Psychology, 8: 375. doi: 10.3389/fpsyg.2017.00375.

Henderson, A. y Mapp, K. (2002). The Impact of School, Family, and Community Connections on Student Achievement. Austin Texas: Advancing Research Improving Education. https://www.sedl.org/connections/resources/evidence.pdf

Ho, E.S. y Lam, T.Y. (2016). Multilevel analyses of families' influence on adolescents literacy performances. International Journal Quantitative Research in Education, 3(1/2), $58-78$.

Johnsen, S. y Corn, A. (2003). SAGES-2: K3 Evaluación Inicial para Estudiantes con Aptitudes Sobresalientes (Educación primaria y secundaria). México: El Manual Moderno.

Kantor, J. R. (1975). Educaction in psychological perspective. The Psychological Record, 25(3), 315 - 323. https://doi.org/10.1007/BF03394321

Karbach, J., Gottschling, J., Spengler, M., Hegewald, K., y Spinath, F. M. (2013). Parental involvement and general cognitive ability as predictors of domain-specific academic achievement in early adolescence. Learning and Instruction, 23, 43-51. https://doi.org/10.1016/j.learninstruc.2012.09.004

Kusumaningrum, F. A., y Alsa, A. (2016). Parents Support, Teachers Support, and Intelligence as Predictors of Mathematics Learning Achievement in Class XI of Yogyakarta Senior High Schools. Mediterranean Journal of Social Sciences, 7(1), 427-434. doi: 10.5901/mjss.2016.v7n1p427

LaRocque, M., Kleiman, I. y Darling, S. M. (2011). Parental involvement: The Missing Link in School Achievement. Preventing School Failure, 55 (3), 115-122. doi: $10.1080 / 10459880903472876$

Lopes, D., Lemos, G.C., Primi, R. y Almeida, L. S. (2015). The relationship between intelligence and academic achievement throughout middle school: The role of students 'prior academic performance. Learning and Individual Differences, 41, 73-78. http://dx.doi.org/10.1016/j.lindif.2015.02.005

Martínez-Otero, V. (2002). Reflexiones psicopedagógicas sobre la inteligencia. PULSO. Revista de Educación, (25), 77-86.

Núñez, J. C., Suárez, N., Rosário, P., Vallejo, G., Valle, A., y Epstein, J. L. (2015). Relationships between perceived parental involvement in homework, student homework behaviors, and academic achievement: differences among elementary, junior high, and 
high school students. Metacognition and learning, 10(3), 375-406. https://doi.org/10.1007/s11409-015-9135-5

OECD (2014). PISA 2012 Technical Report. Available in: http://www.oecd.org/pisa/pisaproducts/PISA-2012-technical-report-final.pdf

Phillipson, S., y Phillipson, S. N. (2012). Children's cognitive ability and their academic achievement: the mediation effects of parental expectations. Asia Pacific Education Review, 13(3), 495-508. https://doi.org/10.1007/s12564-011-9198-1

Poon, K. (2020). The impact of socioeconomic status on parental factors in promoting academic achievement in Chinese children. International Journal of Educational Development, 75, 102175.

Rodríguez, E., Cadenas, M., Borges, Á., y Valadez, D. (2019). Educational responses to students with high abilities from the parental perspective. Frontiers in Psychology, 10: 1187. doi: $10.3389 /$ fpsyg.2019.01187

Sánchez, P. y Acle, G. (2003). Validación y normas para la población del SAGES-2 mexicana. México: UNAM.

Santamaría, P., Arribas, D., Pereña, J., y Seisdedos, N. (2005). EFAI: Evaluación factorial de las aptitudes intelectuales. Madrid: TEA

Santos, M. A., Godás, A., Ferraces, M. J., y Lorenzo, M. (2016). Academic performance of native and immigrant students: a study focused on the perception of family support and control, school satisfaction, and learning environment. Frontiers in Psychology, 7:1560. doi: 10.3389/fpsyg.2016.01560

Sternberg, R. J. (2008). Assessing what Matters. Educational Leadership, 65(4), 20-26.

Sternberg, R. J. (2010). Assessment of gifted students for identification purposes: New techniques for a new millennium. Learning and Individual Differences, 20 (4), 327-336. doi: 10.1016/j.lindif.2009.08. 003.

Sternberg, R. J., Grigorenko, E. L., y Bundy, D. A. (2001). The predictive value of IQ. Merril Palmer Quarterly, 47 (1), 1-41.

Tan, C. Y., Lyu, M., y Peng, B. (2019). Academic Benefits from Parental Involvement are Stratified by Parental Socioeconomic Status: A Meta-analysis. Parenting, 1-47. https://doi.org/10.1080/15295192.2019.1694836

Treviño, E., Valdés, H., Castro, M., Costilla, R., Pardo, C., y Donoso, F. (2010). Factores asociados al logro cognitivo de los estudiantes de América Latina y El Caribe. Santiago: OREALC-UNESCO Santiago y LLECE, 2010. 
Valadez, M.D., López-Aymes, G., Borges, M.A., Zambrano, R., Galán, M. G., Villegas, E.A., y Betancourt, J. (2016). Evaluación de la propuesta de intervenciónA Atención educativa a alumnos y alumnas con aptitudes sobresalientes y talentos específicos implementados en las escuelas primarias de México. En Valadez, M.D., López-Aymes, G., Borges, M. A., Betacourt, J., y Zambrazo, R. (Coords.). Programas para niños con altas capacidades y su evaluación (pp.189-207) México: Manual Moderno.

Valdés, A. y Vera, J. (2012). Hacia una definición del sobresaliente. En A. Valdés y J. Vera (Eds.), Estudiantes intelectualmente sobresalientes (pp. 2-13). México: Pearson.

Yang, X. (2017). Education Equity in Swedish Compulsory School Effects of student background, personal and school characteristics on their academic achievement in PISA 2015. Institutionen För Pedagogik Och Specialpedagogik. Rapport Nr: VT17 IPSPDA184:6 